\title{
Papers
}

\section{Effect of reducing ambulance response times on deaths from out of hospital cardiac arrest: cohort study}

\author{
Jill P Pell, Jane M Sirel, Andrew K Marsden, Ian Ford, Stuart M Cobbe
}

\begin{abstract}
Objectives To determine the association between ambulance response time and survival from out of hospital cardiopulmonary arrest and to estimate the effect of reducing response times.

Design Cohort study.

Setting Scottish Ambulance Service.

Subjects All out of hospital cardiopulmonary arrests due to cardiac disease attended by the Scottish Ambulance Service during May 1991 to March 1998. Main outcome measures Survival rate to hospital discharge and potential improvement from reducing response times.

Results Of 13822 arrests not witnessed by ambulance crews but attended by them within 15 minutes, complete data were available for 10554 (76\%). Of these patients, $653(6 \%)$ survived to hospital discharge. After other significant covariates were adjusted for, shorter response time was significantly associated with increased probability of receiving defibrillation and survival to discharge among those defibrillated. Reducing the 90th centile for response time to 8 minutes increased the predicted survival to $8 \%$, and reducing it to 5 minutes increased survival to $10-11 \%$ (depending on the model used).

Conclusions Reducing ambulance response times to 5 minutes could almost double the survival rate for cardiac arrests not witnessed by ambulance crews.
\end{abstract}

\section{Introduction}

Mortality from coronary heart disease in the United Kingdom is among the highest in the world. ${ }^{1}$ Three quarters of all deaths from myocardial infarction occur after cardiac arrest in the community. ${ }^{2}$ This proportion is even higher in people under 55 years of age, in whom $91 \%$ of cardiac arrest deaths occur out of hospital. $^{2}$ Therefore, the greatest scope to improve survival lies outside hospital.

Currently, the ambulance service has a statutory obligation to arrive at the scene of $50 \%$ of emergency calls within 7 minutes and $90 \%$ within 14 minutes. The government is considering reducing this target to $90 \%$ within 8 minutes. ${ }^{3}$ Another way to reduce delays would be for other first responders, such as firefighters, the police, or community volunteers, to be supplied with "intelligent" defibrillators within a medically controlled system. ${ }^{4}$ The fire service, for example, has more stations than the ambulance service to allow it to meet its more stringent targets- $90 \%$ of vehicles are required to attend the scene of a fire within 5 minutes of the emergency call.

The aims of this study were to determine the association between ambulance response times and survival from out of hospital cardiac arrest and the potential effect on survival of reducing response times.

\section{Methods}

The Scottish Ambulance Service is the sole provider of emergency prehospital ambulance care for the 5.1 million population of Scotland. Since 1988, all front line ambulances have been equipped with an automated external defibrillator, and all ambulance crews are trained in use of defibrillators and in basic life support. Data are collected on all cardiopulmonary resuscitation attempts by ambulance staff. These include the response time (defined as the interval between the emergency call and arrival at the scene), the patient's age and sex, the place of the arrest, the presence of a bystander witness, bystander use of cardiopulmonary resuscitation, whether a defibrillator was used, the make of defibrillator, initial outcome of the resuscitation attempt, and transfer to hospital. The data are collated centrally at the department of medical cardiology, Glasgow Royal Infirmary. Additional information on survival to discharge is obtained for patients admitted to hospital alive.

Since 1991, the presumed cause of arrest has been classified in accordance with the Utstein convention, ${ }^{5}$ whereby arrests are defined as due to cardiac disease if the cause is recorded as either heart disease or unknown. Arrests that were witnessed by the ambulance crew were excluded from our model because reducing response time would be unlikely to affect survival in this group. We also excluded arrests for which data were incomplete.

To estimate the effect of reducing response times, the cohort was randomly divided into two equally sized groups: a training set and a test set. We used stepwise multiple logistic regression on the training set to determine whether a patient received defibrillation.We then did a second stepwise logistic regression analysis on those who received defibrillation to determine the predictors of survival to discharge from hospital. The box lists covariates available for selection in the model. To provide an unbiased assessment of the models derived

\author{
Department of \\ Medical Cardiology, \\ University of \\ Glasgow, Glasgow \\ Royal Infirmary, \\ Glasgow G31 2ER \\ Jill P Pell \\ honorary clinical \\ senior lecturer \\ Jane M Sirel \\ research assistant \\ Stuart M Cobbe \\ Walton professor of \\ medical cardiology \\ Scottish Ambulance \\ Service \\ Headquarters, \\ Edinburgh \\ Andrew K Marsden \\ medical director \\ Robertson Centre \\ for Biostatistics, \\ University of \\ Glasgow \\ Ian Ford \\ professor of statistics \\ Correspondence to: \\ S M Cobbe \\ stuart.cobbe@ \\ clinmed.gla.ac.uk
}

BMJ 2001;322:1385-8 
Variables available in multiple logistic regression models*

Sex

Site (home or other)

Age (continuous variable)

Cardiopulmonary resuscitation from bystander (yes or no)

Bystander witness (present or not)

Time from 999 call to arrival at scene (continuous variable)

Year of arrest

Type of defibrillator

*Interactions were included as required

on the training set, the models were applied to the test set and the observed and predicted number of survivors were compared.

We then assessed the potential impact on survival of a reduction in response time. The effects of reducing the 90 th centile for response time to 8 and 5 minutes were studied in turn. The response times were divided by the 90 th centile and then multiplied by 8 or 5 as appropriate.

Two models were investigated. The first, more conservative, model (A) assumed that the reduction in response times would have no effect on the percentage of patients who were not defibrillated. The second model (B) assumed that a reduction in response time would increase the probability of receiving defibrillation as well as improving survival from defibrillation in those who were defibrillated. Both models assumed that a reduction in response times did not affect survival of patients who were not defibrillated. Survival of these patients was taken to be $1 \%$, which was the figure observed in the dataset.

In model A, the predicted number of survivors who were not defibrillated was taken to be the observed number. Among those who were defibrillated, the predicted number of survivors was taken to be the sum of the estimated conditional probabilities of survival given defibrillation. In model $\mathrm{B}$, the predicted number of survivors was taken to be the sum over all subjects of the estimated probabilities of not being defibrillated multiplied by 0.01 (the expected non-defibrillated survivors) plus the sum over all subjects of the products of the intimated probability of being defibrillated multiplied by the estimated conditional probability of surviving given defibrillation (the expected defibrillated survivors). Full details of both models can be obtained from the authors.

We calculated approximate standard errors for the numbers and percentages of survivors by propagating the error associated with the estimation of the parameters in the logistic regression models through the calculations described above. We assumed that the parameter estimates followed a multivariate normal distribution with mean vector and covariance matrix equal to the maximum likelihood estimates and associated covariance matrix. We generated samples from this distribution, calculated the associated numbers and percentages of survivors, and estimated the standard deviations.

\section{Results}

Of the 21485 cardiopulmonary arrests attended by the Scottish Ambulance Service during May 1991 to March 1998, 16791 (78\%) were due to cardiac disease according to the Utstein convention (figure). The outcome was known in 16509 (98\%) cases. Of these, $14967(91 \%)$ arrests were not witnessed by the ambulance crew. In these cases there was, by definition, a delay between the onset of cardiac arrest, the emergency call, and attendance by the ambulance crew. Response times were recorded for $14866(99 \%)$ of these calls. The current national targets for ambulance response time were met; ambulance crews arrived at the scene within 14 minutes in 13535 (91\%) cases and within 7 minutes in 7568 (51\%).

The response time was over 15 minutes for 1044 (7\%) of the arrests not witnessed by ambulance crews. Despite the long delays in this subgroup, $3 \%$ survived to discharge. Further information was available for 682 of these cases. This indicated that a general practitioner had been in attendance in $419(61 \%)$ cases. We therefore assumed that a high proportion of calls with delays over 15 minutes were not emergency calls from the general public, but general practitioner "urgent" calls for chest pain that were upgraded to emergency calls once a cardiac arrest occurred. Because of the atypical nature and outcome of these calls they were omitted from the remainder of the analysis.

Of the 13822 arrests, which the ambulance crew attended within 15 minutes but did not witness, 10554 $(76 \%)$ had complete predictor and outcome data and therefore could be included in the model. The training set consisted of 5274 arrests. Use of defibrillation was significantly associated with the age and sex of the patient, the presence of a bystander witness, cardiopulmonary resuscitation by a bystander, the site of arrest (all $\mathrm{P}<0.001$ ), and the year in which it took place $(\mathrm{P}<0.05)$. Response time remained a predictor of defibrillation after these factors were adjusted for $(\mathrm{P}<0.001)$

Significant interactions were noted between response time and place of arrest and cardiopulmon-

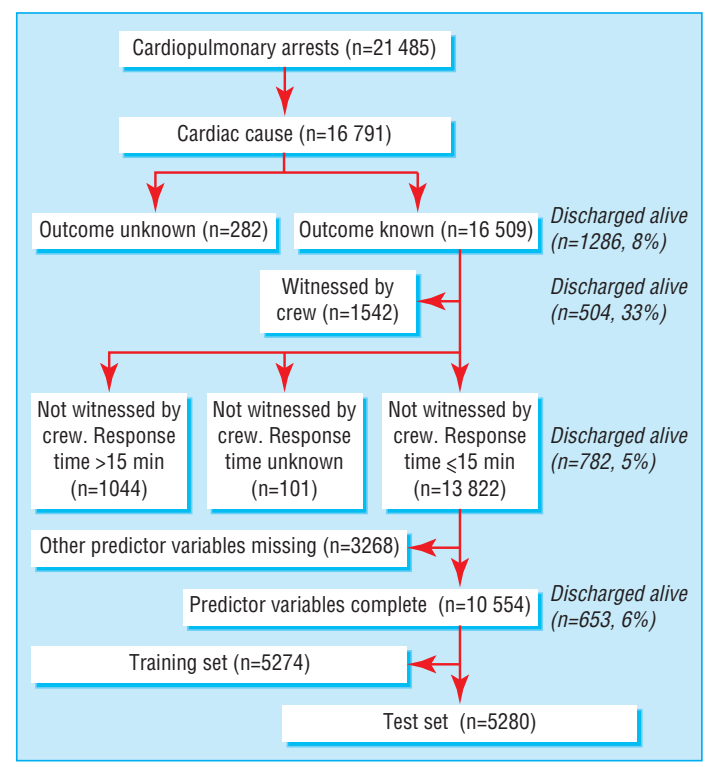

Selection of cardiac arrests for analysis 
Potential increases in survival rates attainable by reducing ambulance response times derived from two models with 5280 arrests

\begin{tabular}{|c|c|c|c|c|c|c|c|c|}
\hline \multirow{3}{*}{$\begin{array}{l}\text { No of } \\
\text { survivors }\end{array}$} & \multicolumn{4}{|c|}{$\mathbf{9 0} \%$ of responses $\leqslant 8 \mathrm{~min}$} & \multicolumn{4}{|c|}{$\mathbf{9 0} \%$ of responses $\leqslant 5 \mathrm{~min}$} \\
\hline & \multicolumn{2}{|c|}{ Model A* } & \multicolumn{2}{|c|}{ Model B† } & \multicolumn{2}{|c|}{ Model A* } & \multicolumn{2}{|c|}{ Model B† } \\
\hline & No $(\%)$ & $95 \% \mathrm{Cl}(\%)$ & No (\%) & $95 \%$ Cl (\%) & No (\%) & $95 \%$ Cl (\%) & No $(\%)$ & $95 \% \mathrm{Cl}(\%)$ \\
\hline Observed & $327(6.2)$ & - & $327(6.2)$ & - & $327(6.2)$ & - & $327(6.2)$ & - \\
\hline Additional & $95(1.8)$ & 1.3 to 2.3 & $116(2.2)$ & 1.7 to 2.7 & $186(3.5)$ & 2.4 to 4.6 & $228(4.3)$ & 3.1 to 5.5 \\
\hline Total & $422(8.0)$ & 7.2 to 8.8 & 443 (8.4) & 7.5 to 9.3 & $513(9.7)$ & 8.4 to 11.0 & 555 (10.5) & 9.1 to 11.9 \\
\hline
\end{tabular}

*Effect on survival of reduced response times in defibrillated patients only.

†Effect on defibrillation of reduced response time plus effect on survival given increased probability of defibrillation.

ary resuscitation from a bystander, and these were included in the model. However, the probability of being defibrillated decreased with increasing response time irrespective of place of arrest and whether a patient received cardiopulmonary resuscitation from a bystander. There were significant interactions between predictor variables other than response time. Full details of these can be obtained from the authors. When the coefficients obtained were applied to the test group of 5280 arrests, defibrillation was predicted in $3560(67 \%)$ patients. The observed number who had defibrillation was 3489 (66\%).

For cardiac arrests in which defibrillation was used, survival to hospital discharge was significantly associated with the presence of a bystander witness, cardiopulmonary resuscitation from a bystander, defibrillator type (all $\mathrm{P}<0.0001$ ), and site of arrest $(\mathrm{P}<0.001)$. Response time remained a predictor of survival after these factors were adjusted for $(\mathrm{P}<0.0001)$. There were no significant interactions. When the coefficients obtained were applied to the test group, $309(9 \%)$ patients were predicted to survive. This compared well with the $312(9 \%)$ who actually survived. Of the 1791 patients who were not defibrillated, $15(1 \%)$ survived.

The table shows that a reduction in the 90th centile response time to 8 minutes resulted in the number of potential survivors increasing from $327(6 \%)$ to 422 $(8 \%$, model A) or $443(8 \%$, model B). If the 90 th centile for response time were 5 minutes, predicted survival increased from $327(6 \%)$ to $513(10 \%$, model A) or 555 (11\%, model B).

\section{Discussion}

Our results show that ambulance response time is a significant independent predictor of both defibrillation and survival to discharge. Currently, ambulances in the United Kingdom are required to respond to $90 \%$ of emergency calls within 14 minutes. Our models suggest that increasing this target to 8 minutes would increase the proportion of potential survivors from $6 \%$ to $8 \%$. Responding to $90 \%$ of calls within 5 minutes would increase the proportion of survivors to $10-11 \%$.

\section{Importance of out of hospital defibrillation}

Survival of out of hospital cardiac arrest in the United Kingdom is up to three times lower than that in some other countries. ${ }^{67}$ The American Heart Association described the "chain of survival" concept in which survival depends on several factors including public awareness of symptoms, early basic life support by bystanders, rapid access to emergency medical services, and prompt defibrillation. ${ }^{89}$ Survival from cardiac arrest can be increased sixfold by providing first line responders with defibrillators. ${ }^{10} 11$
Defibrillation improves survival only in patients with tachyarrhythmic arrests. In our study, 34\% of patients in the test group did not receive defibrillation. The ambulances were equipped with automated defibrillators, which have been shown to administer shocks in $90-94 \%$ of patients in ventricular fibrillation. ${ }^{12}$ Therefore, the reason for not defibrillating in most cases was probably the presence of asystole or pulseless electrical activity (electromechanical dissociation) rather than inappropriate management. Automated defibrillators have the advantage that operators require less training than for manual defibrillators. They have been shown to produce comparable or slightly superior results to manual defibrillators within well established emergency care programmes. ${ }^{13-16}$

\section{Choice of period of analysis}

In patients whose arrests are witnessed by a bystander, the main determinant of survival is the delay from onset of the arrhythmia to defibrillation. ${ }^{9}$ This, in turn, depends on the ambulance response time. Although survival is more closely associated with the time from arrest to defibrillation than with the time from emergency call to defibrillation, no reliable information is available for time of arrest in most out of hospital cardiac arrests. Furthermore, targets for ambulance response times are based on time from the call to arrival at the scene. We therefore used this as the basis of the models.

\section{Effectiveness of reducing response times}

Our figures for improvements in survival from reduced response times should be considered as minimum estimates. A general improvement in response times would result in more people with chest pain being reached by a first responder with a defibrillator before the onset of cardiac arrest. Our data show that survival in such cases is $33 \%$.

It has been estimated that out of hospital resuscitation could reduce deaths from ischaemic heart disease in Scottish residents under 75 years of age by 60 per million. ${ }^{17}$ This equates to a $4 \%$ reduction in total deaths from ischaemic heart disease. ${ }^{17}$ Although this seems to be a relatively low figure, out of hospital resuscitation has made a greater contribution to the fall in mortality from coronary heart disease in developed countries than other established treatments such as antihypertensive drugs, coronary artery bypass grafting, and admission to coronary care units. ${ }^{18}$

Reducing response times would inevitably require additional resources. However, a cost effectiveness study from Ontario suggested that the additional cost would be less if the reduced times were achieved by equipping other first line responders (such as firefighters and the police) with defibrillators. ${ }^{19}$ 


\section{What is already known on this topic}

Three quarters of all deaths from myocardial infarction occur after cardiac arrest in the community

Survival after out of hospital arrest is much lower in the United Kingdom than the United States

\section{What this study adds}

Ambulance response times are independently associated with defibrillation and survival

Decreasing the target for response to $90 \%$ of calls from 14 minutes to 8 minutes would increase survival from $6 \%$ to $8 \%$

A response time of 5 minutes would increase survival to $10-11 \%$

\section{Implications}

Previous studies have shown that the provision of external defibrillators and basic life support training to ambulance crews can improve survival from out of hospital cardiac arrest. ${ }^{17}$ Our results suggest that further improvements could be achieved by reducing response times. We estimated the size of improvement in survival that might have been attained previously had response times been lower. It is more difficult to use the model to predict future improvements because we cannot take into account unmeasurable or unpredictable changes in patient characteristics or service delivery.

We thank the Scottish Ambulance Service and medical records department staff for collecting the data on which these analyses were based and Mr T Aitchison for statistical input.

Contributors: SMC is the coordinator of the Heartstart register and had the original idea for the study. All five authors contributed to the study design and interpretation of the results. JMS did the statistical analyses and IF advised on the statistical methods to be used and their interpretation. JPP wrote the original and revised drafts with feedback from JMS, AKM, IF, and SMC. All authors approved the final version for submission. $\mathrm{SMC}$ is the guarantor.

Funding: British Heart Foundation.

Competing interests: None declared.
1 Rayner M, Mockford C, Boaz A. Coronary heart disease statistics. British Heart Foundation statistics database 1998. Oxford: British Heart Foundation, 1998.

2 Norris RM, United Kingdom Heart Attack Study Collaborative Group. Fatality outside hospital from acute coronary events in three British health districts, 1994-5. BMJ 1998;316:1065-70.

3 NHS Executive. Review of ambulance performance standards: final report of steering group. London: NHS Executive, 1996.

4 Dracup K, Alonzo A, Atkins JM, Bennett NM, Braslow A, Clark LT, et al. The physician's role in minimising pre-hospital delay in patients at high risk for acute myocardial infarction: recommendations from the National Heart Attack Alert Program. Ann Intern Med 1997;126:645-51.

5 Cummins RO, Chamberlain DA, Abramson NS, Allan M, Baskiett PJ, Becker L, et al. Recommended guidelines for uniform reporting of data from out-of-hospital cardiac arrest: the Utstein style. A statement for health professionals from a task force of the American Heart Association, the European Resuscitation Council, the Heart and Stroke Foundation of Canada, and the Australian Resuscitation Council. Circulation 1991;84:960-75.

6 Cobb LA, Weaver WD, Fahrenbruch CE, Hallstrom AP. Communitybased interventions for sudden cardiac death. Impact, limitations and changes. Circulation 1992;85(suppl 1):98-102.

7 Kuisma M, Maala T. Out-of-hospital cardiac arrests in Helsinki: Utstein style reporting. Heart 1996;76:18-23.

8 Cummins RO, Ornato JP, Thies WH, Pepe PE, Billi PE, Seidel J, et al. Advanced life support committee and emergency cardiac care committee of the American Heart Association: improving survival from sudden cardiac arrest: the "chain of survival" concept. Circulation 1991:83:1832-47.

9 Evans T. Cardiac arrests outside hospital. BMJ 1998:316:1031-2.

10 Weaver WD, Copass MK, Hill DL, Fahrenbruch C, Hallstrom AP, Cobb LA. Cardiac arrest treated with a new automated external defibrillator by out-of-hospital first responders. Am J Cardiol 1986;57:1017-21.

11 Stults KE, Brown DD, Schug VL, Bean JA. Prehospital defibrillation performed by emergency medical technicians in rural communities. $N$ Engl J Med 1984;310:219-23.

12 Sedgwick ML, Watson J, Dalziel K, Carrington DJ, Cobbe SM. Efficacy of out of hospital defibrillation by ambulance technicians using automated external defibrillators: the Heartstart Scotland project. Resuscitation 1992;24:73-87.

13 Cummins RO. From concept to standard-of-care? Review of the clinical experience with automated external defibrillators. Ann Emerg Med 1989:18:1269-75

14 Stults KR, Brown DD, Kerber RE. Efficacy of an automated external defibrillator in the management of out of hospital cardiac arrest: validation of the diagnostic algorithm and initial clinical experience in a rural environment. Circulation 1986;73:701-9.

15 Cummins RO, Eisenberg MS, Litwin PE, Graves JR, Hearnes TR, Hallstrom AP. Automatic external defibrillators used by emergency medical technicians: a controlled clinical trial. JAMA 1987;257:1605-10.

16 Weaver WD, Hill D, Fahrenbruch CE, Coppas MK, Martin JS, Cobb LA, et al. Use of the automatic external defibrillator in the management of outof-hospital cardiac arrest. N Engl J Med 1988;319:661-6.

17 Cobbe SM, Redmond MJ, Watson JM, Hollingworth J, Carrington DJ "Heartstart Scotland"-initial experience of a national scheme for out of hospital defibrillation. BMJ 1991;302:1517-20.

18 Beaglehole R. Medical management and the decline in mortality from coronary heart disease. $B M J$ 1986;292:33-5.

19 Nichol G, Laupacis A, Stiell IG, O'Rourke K, Anis A, Bolley H, et al Costeffectiveness analysis of potential improvements to emergency medical services for victims of out-of-hospital cardiac arrest. Ann Intern Med $1996 ; 27: 711-20$

(Accepted 14 March 2001)

\section{Insights on the internet}

The father of a baby in a neonatal intensive care unit was a web page designer. He decided to post a page that described the ongoing medical and nursing care being given to his son and update it regularly. He and his partner told anxious relatives and friends about the page and explained that posting information on it would allow them to concentrate on their son.

"When we first saw Jasper," he wrote, "as well as the ventilator, he had a small tube going down to his stomach to let air escape and for feeding when he reached that stage. He had a line into an artery in his arm which was used to measure blood pressure and heart rate and provide a painless method for taking blood."

He also described possible complications: "The doctors expect that ' 24 weekers' are likely to get a brain haemorrhage at some stage. These seem to be categorised small, medium, or large. Jasper has developed a medium one which although it isn't life threatening, needs to be kept an eye on. It could just melt away, or it could cause future complications that have been anticipated."
Not only did the website benefit the baby's relatives, it had an unexpected advantage for medical staff: it provided documentation of what the parents understood about their son's condition. We thus had direct feedback about our communication to the parents and about which facts had been misinterpreted. This allowed conversations with the parents to be tailored specifically to their understanding of their son's condition. The site was also useful for staff on different shifts because it helped to establish what had been discussed previously.

Feedback is essential; it helps to improve both clinical and non-clinical skills, but it can be hard to obtain feedback on areas such as communication. We should make the most of feedback regardless of where it comes from and keep our eyes open to opportunities that may give us a special insight into the minds of our patients and their families.

Sarah J Bridges specialist registrar in paediatrics, Southmead Hospital, Bristol 